\title{
184. On the Mechanism of Formation of Step-Faults in Sand Layers-a Possible Analogy with Slip Bands in Deformed Metallic Crystals.
}

\author{
By Torahiko Terada and Naomi Miyabe. \\ Earthquake Research Institute.
}

(Comm. by T. Terada, M.I.A., Dec. 12, 1927.)

If one of the side walls of a rectangular box in which a horizontal layer of fine dry sand is deposited, is made to recede outwards, the sliding motion of the sand mass towards the receding wall takes place

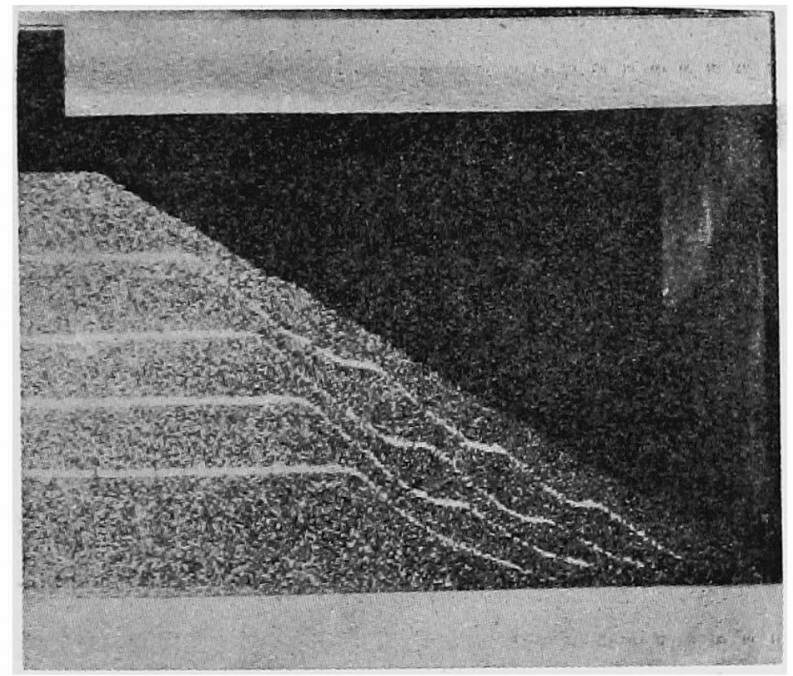

Fig. 1. in discrete steps such as to form a set of step-faults (Fig. 1) quite similar in appearance to those frequently observed in geotectonic structures, or in the cases of land-slips in smaller scale. The mechanism of formation of these faults in sand was studied in some details.

A rectangular vessel, $50 \mathrm{~cm}$. $\times 20 \mathrm{~cm} . \times 20 \mathrm{~cm}$. was employed, of which the longer lateral walls were made of thick glass plates, and the end walls made of wood were capable of regulated motion in the direction perpendicular to it, i.e. in the direction of the length of the vessel, by means of a suitable screw. The sand layer was marked by three or four horizontal lines of white sand arranged along the glass wall such that by taking successive photograms of this marked side during the motion of the receding end wall, the general mode of deformation of the sand mass could be studied.

The slip line, i.e. the trace of the slip plane, obtained in this manner is generally curved, the inclination to the horizontal increasing upwards. Some authorities ${ }^{1)}$ state that the curvature of the slip

1) A. Strecke, Bauingenieur, 7 (1926), Hft. 1 and 2. 
line obtained in some experiments is due to the accidental vertical component of motion of the receding wall and should disappear if the vertical wall is moved in exactly horizontal direction. The present result is, however, not necessarily in contradiction with the above statement, as the case here studied is the dynamical one in which the sand mass is in a continuous motion, whereas the previous investigations deals mainly with the statical case pertaining to the very beginning of the slip motion.

The curve of the slip line may fairly be represented by a formula

$$
y=k x^{n}
$$

where $x$ is the vertical height of the point of the curve from the bottom, $y$ the horizontal distance of the point from the moving wall and $k, n$ some constants. ${ }^{1)}$ The lower end of the curve always coincides with the foot of the receding wall. While the wall is moving, the value of $k$ and $n$ are subjected to characteristic variations, meanwhile preserving the form expressed by the above formula.

Experimenting with loosely packed sand on one hand and closely packed one on the other hand, it was ascertained that the corresponding value of $k$ is generally larger for the loose one than for the close one, as might have been expected, since in the ordinary theory of earth pressure the angle of inclination of the slip line (considered as straight) is nearly twice the angle of repose. The curvature of the slip line may then be explained if we consider that by the receding motion of the wall, the sand mass between the wall and the slip plane is more disturbed in the lower layer than in the upper.

The gradual change in the trend of a slip plane may be illustrated by Fig. 2. As the wall recedes, $k$ increases corresponding to the gradual loosening of packing. A limit is, however, reached beyond which $k$ cannot be increased. Then, a new slip line starts from the foot of the wall and the former slip plane is arrested. The second slip plane repeats a similar mode of variation as that of the first until the limiting slope is again attained, resulting in the formation of the third slip plane and so on.

In this manner, the interval between successive faults is determined ( 1 ) by the difference between the maximum and minimum "coefficient of friction" and (2) by the height of the sand layer.

1) The uppermost part of the slip line must be excluded, as that part is disturbed by the subsequent destruction of the fault scarp. 


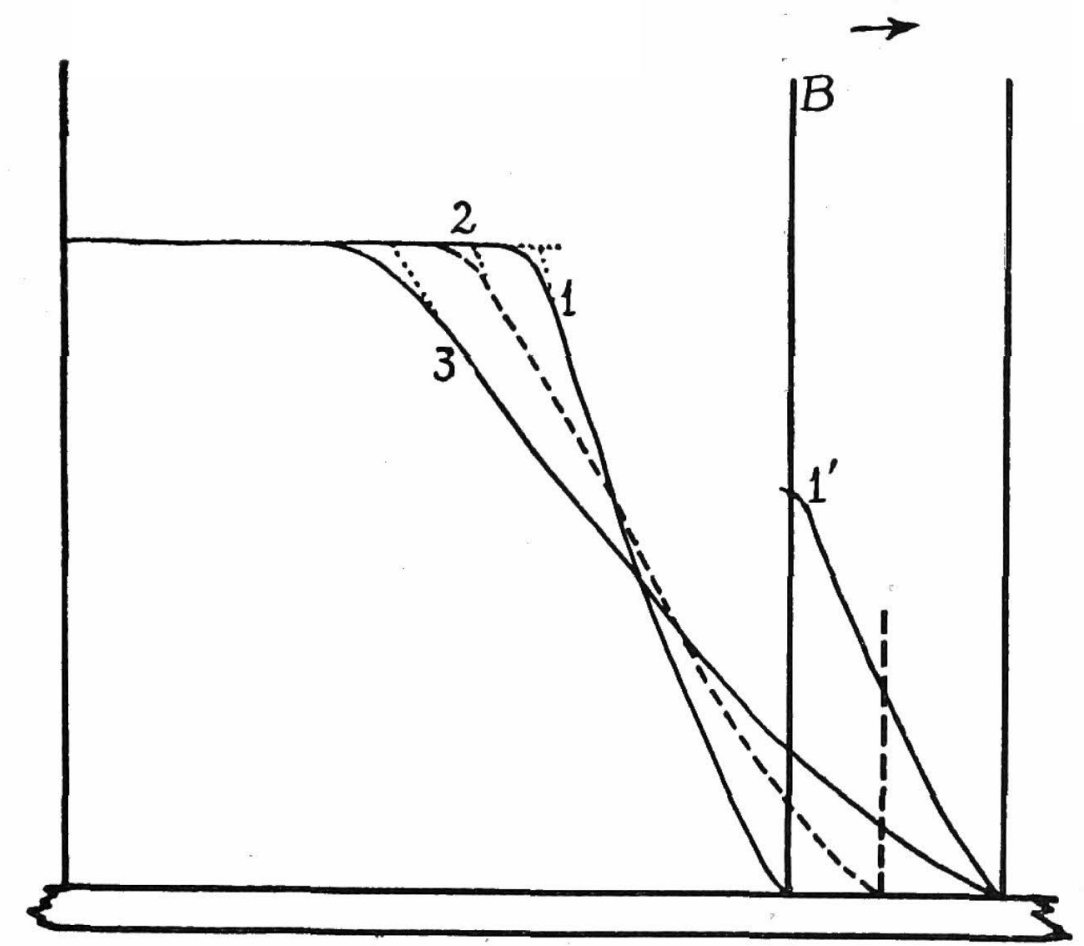

Fig. 2. B : moving wall.

1: initial slip plane.

3 : limiting position of 1 st. slp. pl.

$\rightarrow$ : direction of motion.

2: intermediate slp. pl.

$1^{\prime}$ : initial position of 2nd. slp. pl.

When the movable wall is pushed inwards so as to laterally compress the sand layer, a fan-shaped system of thrust faults (Fig. 3) ensues, such as already studied experimentally by Cadell ${ }^{1)}$ with the

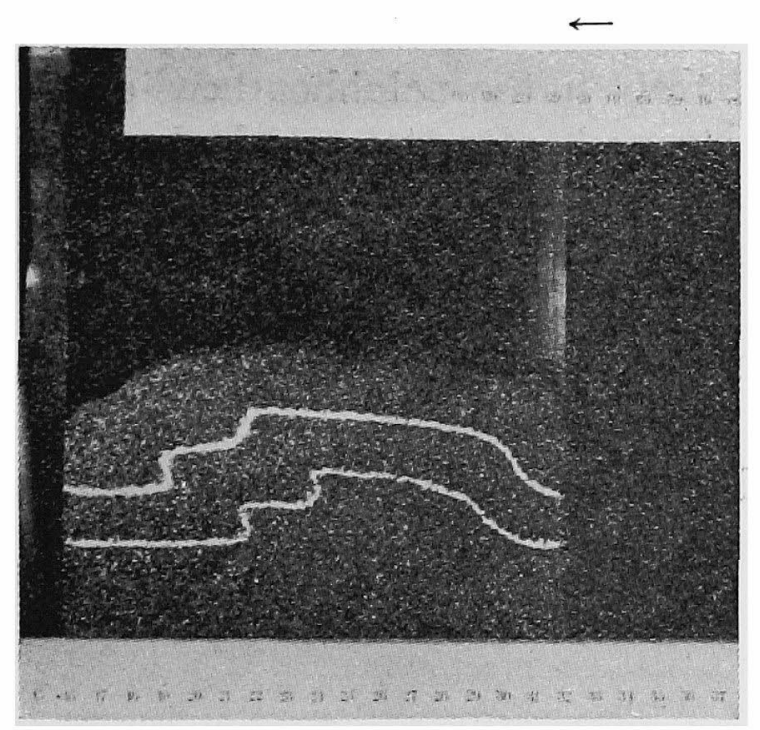

Fig. 3. Right side wall pushed in. purpose of explaining some characteristic geological structure met with in Scotland. The mechanism is quite similar to the above case.

The wedge-like portion of sand between the receding wall and the slip plane is found to be generally elongated in the horizontal direction, while it is contracted vertically, i.e. as if deformed by an apparent horizontal tension. Between this wedge-like mass which behaves apparently as an imperfectly elastic mass and that mass lying behind the slip plane

1) H. M. Cadell, Trans. Roy. Soc. Edinburgh, 35 (1888) 337. 
which behaves as a rigid body, there exists a distinct intermediate zone which behaves in some sense as a viscous fluid. In this last zone the deformation of the whole mass is localized or concentrated. Fig. 4 shows this zone in a magnified scale.

The results of the above experiments which were carried out in connection with a geophysi-

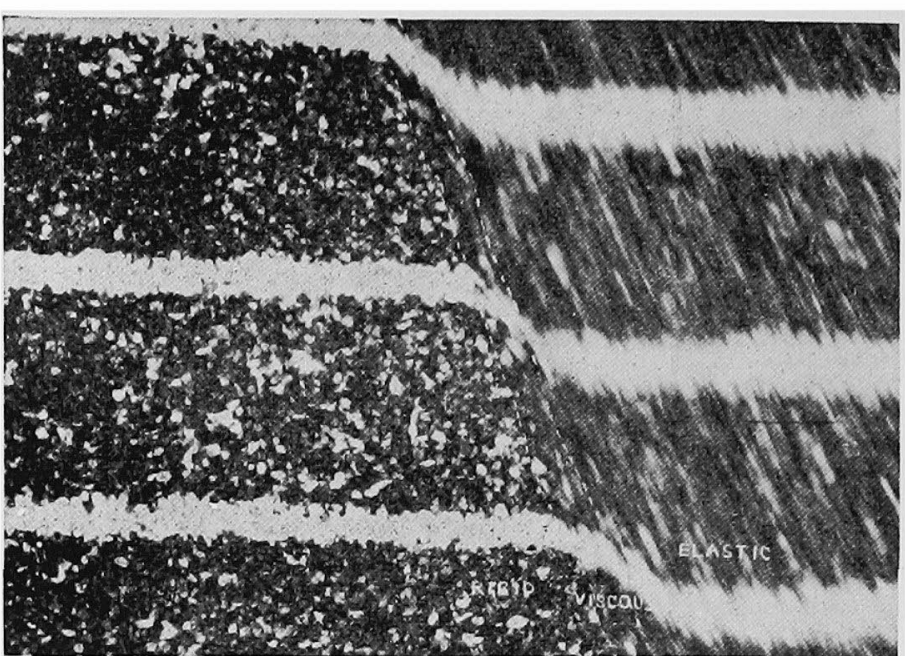

Fig. 4. Rigid $|\rightarrow|$ Elastic Viscous cal investigation, will be of some interest for those who are concerned with investigations on the strength of material in general. Moreover, the above phenomenon suggests some formal analogy of it with the formation of slip bands in deformed metallic crystal.

The mechanism of "self-stopping" of slip bands is still obscure and especially the remarkable quasiperiodicity of the bands has not yet received any plausible explanation. It is probable, as is generally believed, that the stopping of slip is brought about by the hardening of that zone caused by molecular derangement. The change of the boundary condition due to the formation and stopping of a slip plane will result in some concentration of strain in the neighbourhood of the primary slip band. Moreover, the strength of metal may be lessened in some adjacent zone as another consequence of the same derangement. Though it will be premature to propose here any definite working model, the above case of the step faults in sand mass may serve at least as a hint for those who are engaged in the investigations for elucidating the true mechanism of the slip bands.

The details of the results of investigations will be published in Vol. IV of the Bulletin of the Earthquake Research Institute. 